\title{
ICaRO: a new cosmic ray detector at Izaña Atmospheric Observatory
}

\author{
Juan José Blanco Avalos, ${ }^{a, *}$ Juan Ignacio García-Tejedor, ${ }^{a}$ Óscar \\ García-Población, ${ }^{a}$ Sindulfo Ayuso de Gregorio, ${ }^{a}$ Iván Vrublevskyy, ${ }^{a}$ \\ Alejandro López-Comazzi, ${ }^{a}$ Almudena Gomis Moreno, ${ }^{b}$ David Moure \\ García, ${ }^{b}$ Emilio Cuevas, ${ }^{c}$ África Barreto Velasco ${ }^{c}$ and Ramón Ramos ${ }^{c}$ \\ ${ }^{a}$ Space Research Group, University of Alcalá, Spain \\ ${ }^{b}$ Instituto Geográfico Nacional, Spain \\ ${ }^{c}$ Observatorio Atmosférico de Izaña, Agencia Estatal de Meteorología, Spain \\ E-mail: juanjo.blanco@uah.es
}

A twin detector of ORCA, the cosmic ray detector operating at Juan Carlos I Spanish Antarctic Base, is foreseen to be installed at Izaña Observatory (IZO) during the second part of 2021. IZO belongs to the State Meteorological Agency of Spain (AEMET) and it is located at the top of a mountain plateau in Teide volcano at Tenerife Island $\left(28^{\circ} 18^{\prime} \mathrm{N}\right.$, $16^{\circ} 29^{\prime} \mathrm{W}, 2400 \mathrm{~m}$ a.s.l.) at vertical cut-off rigidity of $11.5 \mathrm{GV}$. ICaRO (Izaña Cosmic Ray Observatory) is composed of a BF3-based 3NM64 (ICRO) and a 3 bare BF3 counters (ICRB). The neutron monitor is complemented by a muon telescope sharing a common room in a single stack. The muon telescope follows the MITO approach, and thus is composed of two scintillator layers, Top and Bottom. It is able to provide muon counting rate and muon impact points on the scintillator layers. MITO's layers are $1.365 \mathrm{~m}$ apart with the two BF3 sets, ICRO and ICRB, in between. As such, the lead surrounding ICRO acts as filter for particles traversing throughout Top and Bottom. ICaRO will provide counting rates of neutrons in two energy thresholds, muon counting rate and muon incoming directions throughout the detector volume.

$3^{\text {tth }}$ International Cosmic Ray Conference (ICRC 2021)

July 12th - 23rd, 2021

Online - Berlin, Germany

${ }^{*}$ Presenter 


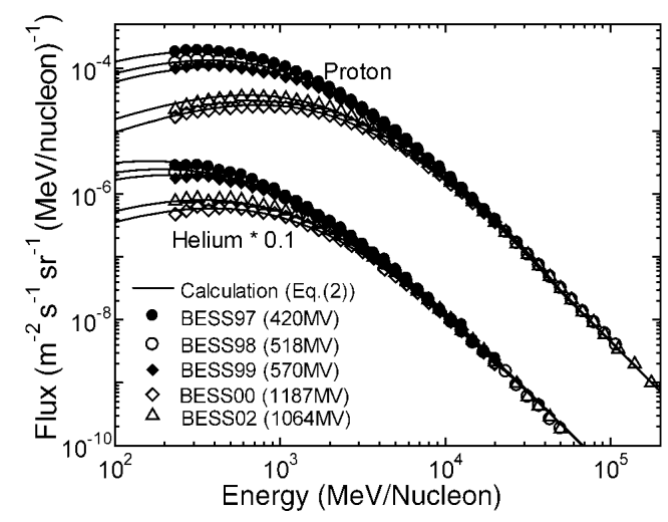

Figure 1: Primary cosmic ray at the top of atmosfere [1].

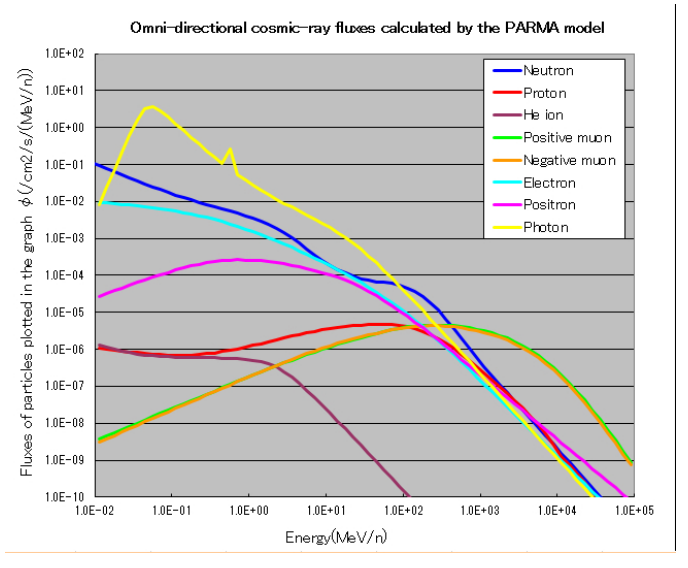

Figure 2: Secundary cosmic rays at the height of $2400 \mathrm{~m}$ at Tenerife Island (EXPACS EXcel-based Program for calculating Atmospheric Cosmic-ray Spectrum https: //phits.jaea.go.jp/expacs/.

\section{Introduction}

Cosmic rays $(\mathrm{CR})$ are atomic nuclei accelerated to relativistic energies in explosive processes in stars, supernovae and supernova remnants, and active galaxy nuclei. From their sources, they travel through the galaxy, cross the heliosphere, and finally reach Earth bringing information about the sources that produce them and the interstellar and interplanetary medium they pass through. Once they arrive at Earth, they interact with the atmosphere producing a cascade of secondary particles (secondary cosmic rays) that can be detected by ground-level instruments. Figure 1 depicts the proton and helium components in $\mathrm{CR}$ at the top of the atmosphere, i.e., before they interact with the molecules of the atmosphere and the production of secondaries begins. The figure shows observations from 1997 to 2002 , i. e. from solar minimum to solar maximum during the solar cycle 23. The observed differences in primary flux between 0.1 and $100 \mathrm{GeV} /$ nucleon are caused by solar cycle evolution. Figure 2 shows the simulated spectrum of secondary cosmic rays arriving at the Canary Islands at an altitude of $2.400 \mathrm{~m}$. The spectrum has been simulated using the PARMA model [1]. Two of the most important secondary are neutrons and muons. The former because of their abundance, as shown in the model results and the latter because of their penetration capability. Detectors capable of recording the flux of these two populations at ground level and at the energies at which the solar activity is able to modulate their flux are of crucial importance to study solar activity.

A neutron monitor is an instrument generally located at ground level designed to measure secondary neutrons produced by the interaction of solar CRs and energetic particles with molecules in the atmosphere. The height at which the detector is located and the vertical cutoff rigidity of its geographic location determines the flux and sensitivity to the energy of the solar RCs and solar energetic particles [2].

A muon telescope follows a different detection technique. They are based on the ion- 
ization produced by charged particles passing through a ionization-sensitive surface and are usually composed of several sensitive planes (typically two) stacked together to form a true telescope. They usually operate by determining the coincidence combination of two detector elements in different planes, which makes it possible to determine the incidence direction of muons [3].

The two instruments are complementary since neutron monitors are sensitive to CR energies from $0.5 \mathrm{GeV}$ to $50 \mathrm{GeV}$ and the muon telescopes show a relevant response to telescopes have a relevant response to $\mathrm{CR}$ from $10 \mathrm{GeV}$ to several hundred $\mathrm{GeV}$.

\section{The Earth as a global detector}

The location of a given detector, whether it is a neutron monitor or a muon telescope, determines the minimum energy needed for a cosmic ray to produce an observable cascade at the detector position. The magnitude that characterizes this is the vertical cutoff rigidity of the station and is typically measured in GV. This makes the global coverage of comparable detectors desirable, to ensure detection at different energy thresholds and to cover different regions of the sky. The neutron monitor database (NDMB), which provides access to neutron monitor measurements from stations around the world, is an example of this. NMDB provides access to real-time as well as historical data (https://www.nmdb.eu/). As shown in Figure 3, which depicts the position of many of neutron monitors around world, there are large uncovered regions, especially in central Africa and in the oceans. The Canary Islands could partially cover such gaps.

Solar activity produces temporal variations in the cosmic ray flux detected at ground level stations. These variations have characteristic times ranging from the 11 years of the solar cycle through variations of several days, such as Forbush decreases (FD), to hours, such as the growth phase of a ground level enhancement (GLE). During a FD, the neutron monitor counting rates show a fast decrease as long as several hours, followed by a gradual recovery lasting several days. This event happens when an interplanetary structure such as an ICME (interplanetary counterpart of a coronal mass ejection) reaches Earth's orbit. A FD is observed by the whole neutron monitor network and the depth of a particular FD depends on the location and height of each neutron monitor. Figure 4 shows the observation of a FD on June 2015 (second panel in the figure). This FD is also observed by TRISTAN [4] which is a ground base instrument sensitive to atmospheric muons produced by cosmic rays (Figure 4, first panel). A strong geomagnetic storm is also observed in coincidence with the FD and the arrival of a complex ICME at Earth's orbit. Among the stations presented is CaLMa, the Castilla-La Mancha neutron monitor [5].

In contrast, the accelerating conditions of particles in the solar photosphere-corona may be sufficient to accelerate solar particles up to energies of several $\mathrm{GeV} /$ nucleon and thus be sufficient, to be detected as a sharp increase in neutron monitor counts. Figure 5 shows GLE71 detected in May 2012. On these dates CaLMa was not fully operational and did not supply data to the NMDB. These two figures demonstrate that both neutron monitors and muon telescopes allow the study of solar activity by observing the variations of detected flux both of neutrons and muons. 

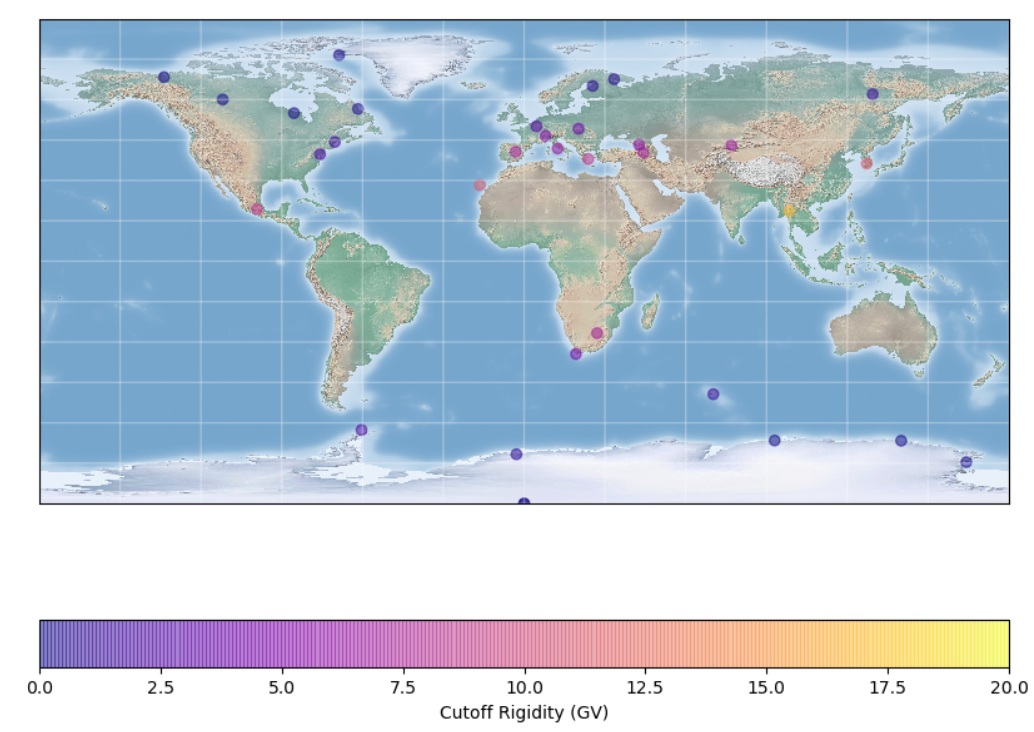

Figure 3: Neutron monitor global distribution. Color bar shows vertical cutoff rigidity range.

\section{Why the Canary Islands?}

The Canary Islands provide coverage of an area currently uncovered by the NMDB, a location with high vertical cutoff rigidity and more than $2000 \mathrm{~m}$ asl as observation point. In a recent work, Artamonov et al. [6] have determined the expected noise signal quality for different neutron monitors during the observation of a solar neutron event and pointed out two gaps that they believe need to be filled. One of these gaps could be filled with a neutron monitor at the Canary Islands.

During the explosive processes that take place in a flare, the accelerated nuclei, mainly protons, interact with the medium and produce neutrons by collision with atoms in the lower layers of the solar atmosphere. The produced neutrons can easily escape from the Sun unaffected by the magnetic fields they encounter on their way to the Earth. Once in the atmosphere, their flux is strongly attenuated and their observation is clearer the higher the height at which the observatory is located. Another factor to take into account is the magnetic rigidity of the station. The higher the rigidity, the more difficult is the arrival of neutrons produced by the protons coming from the same flare, since these are strongly affected by the magnetic field, the clearest neutron signal will be that dues to solar neutrons. These neutrons bring us closer to the conditions under acceleration occurs in solar flares. So two ingredients, height and magnetic rigidity make a site suitable for the observation of solar neutrons. Both of them are fulfilled by Canary Islands. 


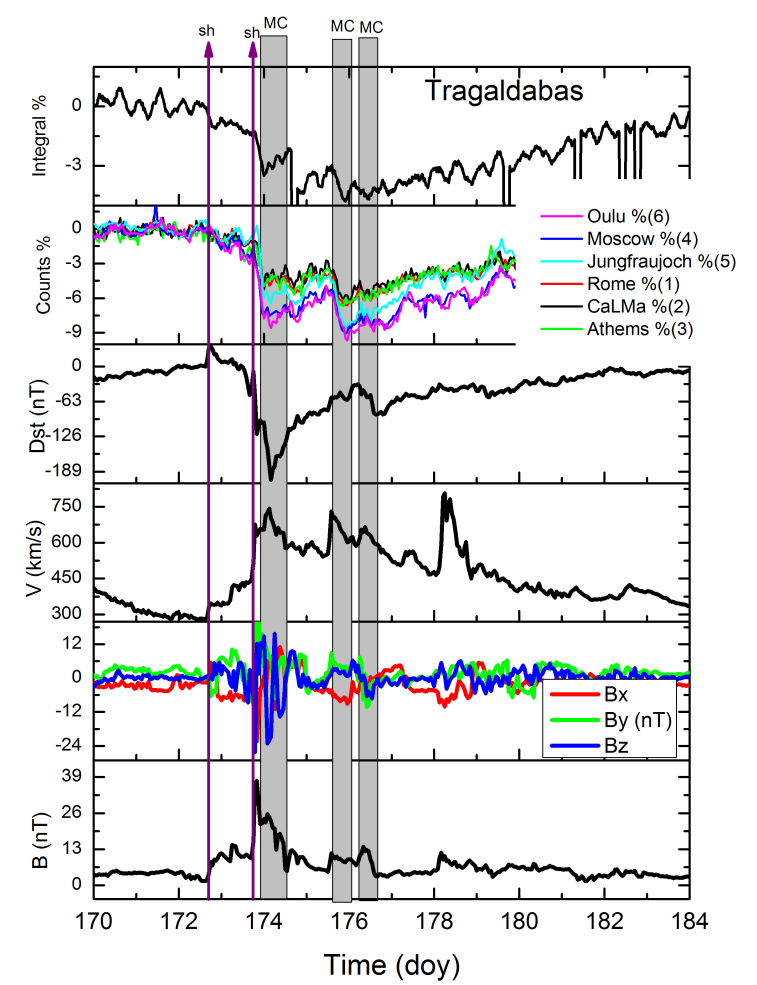

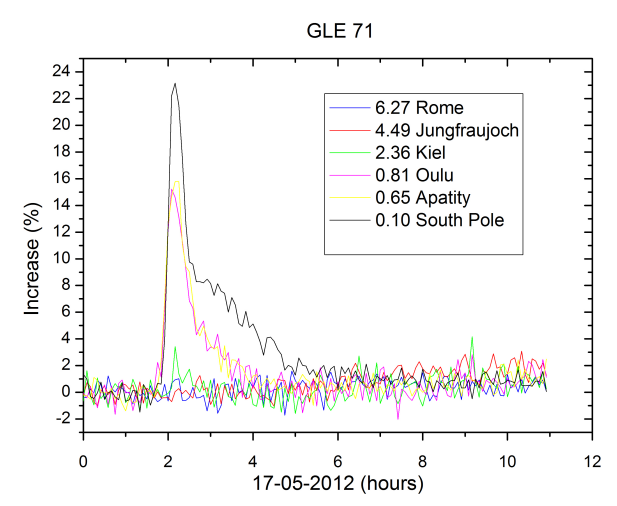

Figure 5: GLE 71 as it is observed by the neutron monitor network.

Figure 4: FD observed by a neutron monitor network and TRISTAN instrument on June 2015 $[4]$

\subsection{Izaña Atmospheric Observatory (IZO)}

The Izaña Observatory, managed by the Izaña Atmospheric Research Centre (AEMET), is at the island of Tenerife at $28^{\circ} 18^{\prime} N, 16^{\circ} 29^{\prime} W, 2373 \mathrm{~m}$ a.s.l (Figure 6). The observatory is located on the top of a mountain plateau. IZO is normally above a temperature inversion layer, generally well established over the island, and so free of local anthropogenic influences. The IZO conducts observations and research related to atmospheric constituents that are capable of forcing change in the climate of the Earth (greenhouse gases and aerosols), and may cause depletion of the global ozone layer, and play key roles in air quality from local to global scales. The IZO has contributed to the World Meteorological Organization (WMO) Global Atmosphere Watch (GAW) Programme (https://public.wmo.int/en) since its establishment in 1989. GAW integrates a number of WMO research and monitoring activities in the field of atmospheric environment. The main objective of GAW is to provide data and other information on the chemical composition and related physical characteristics of the atmosphere and their trends. The IZO also contributes to the Network for the Detection of Atmospheric Composition Change (NDACC). NDACC is an international network for monitoring atmospheric composition using remote measurement techniques [7]. Originally, 


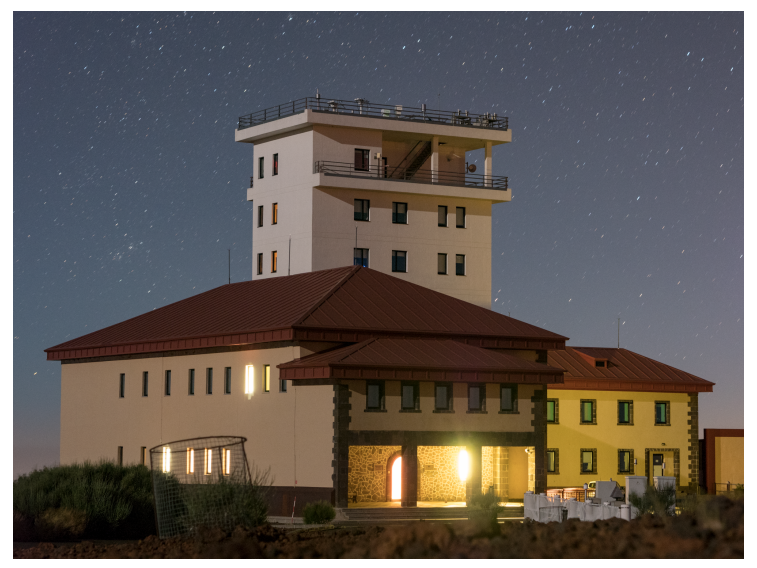

Figure 6: Izaña Atmospheric Research Center.

NDACC was created to monitor the physical and chemical changes in the stratosphere, with special emphasis on the evolution of the ozone layer and the substances responsible for its destruction known as Ozone Depleting Substances. The current objectives of NDACC are to observe and to understand the physicochemical processes of the upper troposphere and stratosphere, and their interactions, and to detect long-term trends of atmospheric composition. A detailed description of the Izaña Observatory facilities and the observation and research programs is shown in [8].

\subsection{Güimar Geomagnetic Observatory (GGO)}

The National Geographic Institute currently has two observatories. One of them is located in the municipality of San Pablo de los Montes (Toledo) and the other in Güímar (Tenerife). The Güimar Geomagnetic Observatory (GGO) is located in Tenerife Island at $28^{\circ} 19^{\prime} 15^{\prime \prime} N, 16^{\circ} 26^{\prime} 26^{\prime \prime} \mathrm{W}$ and $868 \mathrm{~m}$ asl. Its main objective is to continuously and accurately record the values of the geomagnetic components and the total magnetic field.

To measure the Earth's magnetic field, they have two types of instrumentation. The first group consists of variometers, which continuously and automatically record the elements of the geomagnetic field and require installation in thermally controlled environments and on a completely stable platform. A second group is formed by absolute instruments, which are used to measure the geomagnetic field with periodic observations by an observer, and which allow the values measured by the variometers to be scaled.

By processing the data obtained in the observatories, it is possible to determine the indices of geomagnetic activity, the average hourly, daily, monthly and annual values, as well as the annual variation of the geomagnetic elements, and to use them to prepare the Geomagnetic Yearbooks.

\section{Izaña Cosmic Ray Observatory (ICaRO)}

The Izaña Cosmic Ray Observatory (ICaRO) is ORCA's twin instrument. ICaRO will measure neutron and muon counting rates and muon incident direction thanks to two 


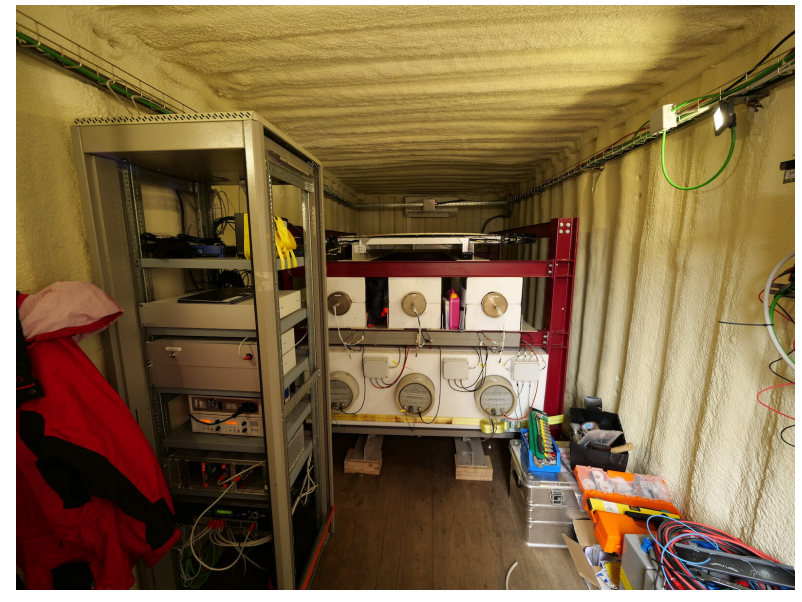

Figure 7: Inside ORCA in a maritime container at Juan Carlos I Station (Livingston Island).

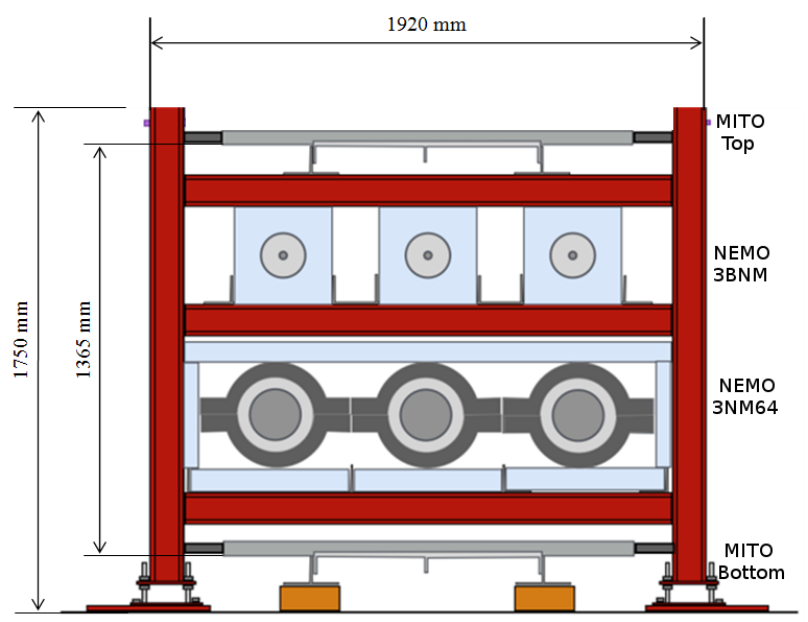

Figure 8: NEMO and MITO scheme.

instruments, NEMO and MITO [9, 10]. Both instruments share a common structure which keeps the relative position of the different elements of NEMO and MITO. A picture of the distribution of the detectors is shown in Figure 7 and a scheme of the distribution of the elements of NEMO and MITO is shown in Figure 8. The first set (NEMO-3NM64) is composed of BP-28 counters, and follows the NM64 standard [11], i. e. an outer reflector made of polyethylene, with lead producers in the form of lead rings around the moderator, a moderator also of polyethylene and the counter tube surrounded by the moderator. The second set is composed by three bare, i. e. without lead producer or polyethylene, LND2061 counters (NEMO-3BNM). They are piled on top of each other, being NEMO-3NM64 below NEMO-3BNM, with the two scintillators which conform MITO on the top and at the bottom to complete the array (Fig. 8).

The Muon Impact-Tracer Observer (MITO) [10] is a telescope made by a stack of two (MITO-Top and MITO-Bottom) BC-400 organic scintillators $(100 \mathrm{~cm} \mathrm{x} 100 \mathrm{~cm}$ x $5 \mathrm{~cm}$, poly-vinyl-toluene with $65 \%$ anthracene). Four photomultipliers tubes (PMTs) are coupled to each scintillator by means of a pyramidal light guide. Each PMT collects the light reaching the lateral surface and generates a pulse which is related to the distance between the particle impact point and the corresponding lateral surface of the scintillator. Particle track is rebuilt by combining of computed impact point at Top and Bottom.

\section{Conclusions}

ICaRO is an instrument designed to monitor secondary cosmic rays. It will be installed at Izaña Observatory (AEMET) $\left(28^{\circ} 18^{\prime} N, 16^{\circ} 29^{\prime} W, 2400 \mathrm{~m}\right.$ a.s.l.) at vertical cut-off rigidity of $11.5 \mathrm{GV}$.

ICaRO is a direct heritage of ORCA. It will provide neutron counting rates at two different thresholds, high energy muon counting rate, low energy muons, charged particles (electron mostly) counting rates and arrival directions for particles passing through the entire detector. 
ICaRO, IZO and GGO will form a complete set of instruments for the study of the effect of cosmic rays on the atmosphere and the monitoring of solar and geomagnetic activity.

\section{Acknowledgements}

Data of neutron monitors has been downloaded from NMDB page: http://www.nmdb. eu/nest/. Dst index from http://wdc.kugi.kyoto-u.ac.jp/dstdir/. Spacecraft data from https://cdaweb.gsf c.nasa.gov/WebServices/. Authors thank to Dr. Juan Garzón for providing TRISTAN data. Thanks to the project PID2019-107806GB-I00, funded by Ministerio de Ciencia e Innovación.

\section{References}

[1] Tatsuhiko Sato, Hiroshi Yasuda, Koji Niita, Akira Endo, and Lembit Sihver. Development of PARMA: PHITS-based Analytical Radiation Model in the Atmosphere. Radiation Research, 170(2):244 - 259, 2008.

[2] John A. Simpson. The cosmic ray nucleonic component: The invention and scientific uses of the neutron monitor - (keynote lecture). Space Science Reviews, 93(1):11-32, Jul 2000.

[3] Marc L. Duldig. Muon observations. Space Science Reviews, 93(1):207-226, Jul 2000.

[4] J. A. Garzón. Trasgos: Towards a new standard for the regular measurement of cosmic. Phys. Atom. Nuclei, 83:453-462-174, 2020.

[5] José Medina, Juan J. Blanco, Oscar García, Raúl Gómez-Herrero, Edwin J. Catalán, Ignacio García, Miguel A. Hidalgo, Daniel Meziat, Manuel Prieto, Javier RodríguezPacheco, and Sebastián Sánchez. Castilla-la mancha neutron monitor. Nucl. Instrum. Methods Phys. Res. A, 727:97-103, 2013.

[6] A. A. Artamonov, G. A. Kovaltsov, A. L. Mishev, and I. G. Usoskin. Neutron monitor yield function for solar neutrons: A new computation. Journal of Geophysical Research: Space Physics, 121(1):117-128, 2016.

[7] M. De Mazière, A. M. Thompson, M. J. Kurylo, J. D. Wild, G. Bernhard, T. Blumenstock, G. O. Braathen, J. W. Hannigan, J.-C. Lambert, T. Leblanc, T. J. McGee, G. Nedoluha, I. Petropavlovskikh, G. Seckmeyer, P. C. Simon, W. Steinbrecht, and S. E. Strahan. The network for the detection of atmospheric composition change (ndacc): history, status and perspectives. Atmospheric Chemistry and Physics, 18(7):4935-4964, 2018.

[8] E. Cuevas, C. Milford, J. J. Bustos, O. E. García, R. D. García, A. J. Gómez Peláez, C. Guirado Fuentes, C. Marrero, N. Prats, R. Ramos, A. Redondas, E. Reyes, P. P. Rivas Soriano, S. Rodríguez, P. M. Romero Campos, C. J. Torres, M. Schneider, M. Yela, J. Belmonte, R. del Campo Hernández, F. Almansa, A. Barreto, C. López Solano, 
S. Basart, E. Terradellas, E. Werner, S. Afonso, C. Bayo, A. Berjón, V. Carreño, N. J. Castro, N. Chinea, A. M. Cruz, M. Damas, F. de Ory Ajamil, M. I. García, V. Gómez Trueba, C. Hernández, Y. Hernández, B. Hernández Cruz, S. F. León Luis, R. López Fernández, J. López Solano, F. Parra, E. Rodríguez, M. Rodríguez Valido, C. Sálamo, E. Sanromá, D. Santana Díaz, F. Santo Tomás, E. Sepúlveda, and E. Sosa. Izaña atmospheric research centeractivity report 2017-2018. Technical report, Joint publication of State Meteorological Agency (AEMET)and World Meteorological Organization (WMO), WMO/GAW Report No. 247, 2019.

[9] J.J. Blanco, O. García Población, J.I. García Tejedor, J. Medina, M. Prieto, A. LópezComazzi, S. Ayuso, and R. Gómez-Herrero. A new neutron monitor at the Juan Carlos I Spanish Antarctic Station. In P. Desiati, T. Gaisser, and A Karle, editors, 36th International Cosmic Ray Conference (ICRC2019), volume 1 of Proceedings of Science, July 2019.

[10] Sindulfo Ayuso, Juan J. Blanco, Juan I. García-Tejedor, Raúl Gómez-Herrero, Iván Vrublevskyy, Óscar García-Población, and José Medina. Mito: a new directional muon telescope. J. Space Weather Space Clim., 11:13, 2021.

[11] M.A. Shea and D.F. Smart. Fifty years of cosmic radiation data. Space Sci. Rev., 93(1):229-262, Jul 2000. 